\title{
Biochemische Grundlagen, Photosensibilisatoren und Lichtquellen
}

\section{Fritsch}

\author{
Biochemical Basics, Photosensitizers and Light Sources
}

Zusammenfassung

Die Fluoreszenzdiagnostik und die photodynamische Therapie werden in das dermatologische Leistungsspektrum aufgenommen. In diesem Artikel sind die biochemischen und physikalischen Grundlagen dieser innovativen Techniken aufgeschlüsselt. Weiterhin werden die Wertigkeit der Photosensibilisatorvorstufe ALA-Methylester herausgestellt sowie die verschiedenen auf dem Markt verfügbaren Lichtquellen beleuchtet.

\section{Biochemische Grundlagen}

\section{Physikalischer Hintergrund der Fluoreszenz}

Die Fluoreszenz ist die Grundlage der Fluoreszenzdetektion von Tumoren. Unter Fluoreszenz wird die Lichtausstrahlung durch die Emission von Atomen oder Molekülen verstanden, die durch Energieabsorption stimuliert werden.

Lichtabsorption: Wenn Licht auf ein Medium trifft, dann wird ein Teil des Lichtes reflektiert, ein Teil wird absorbiert und ein Teil durchdringt das Medium. Die absorbierten Lichtstrahlen werden in Hitze oder andere Energieformen umgewandelt. Das angeleuchtete Molekül - in unserem Fall Porphyrin - wird in einen höher energetischen Zustand gebracht.

Lichtemission: Elektromagnetische Strahlung wird frei (Emission), wenn das angeregte Molekül in seinen Grundzustand zurückfällt. All diese Emissionsprozesse werden unter dem Terminus Lumineszenz zusammengefasst. Die Strahlung oder die Emission eines Moleküls kann leicht ausgelöst werden, wenn die
Abstract

Fluorescence diagnosis and photodynamic therapy are innovative strategies which are frequently included into the dermatological spectrum. Here, the biochemical and physical background of these encouraging techniques is clarified. In addition, the valuability of the photosensitizer's prodrug ALA-methylester is elaborated as well as the features of the available light sources are discussed.

Menge des Moleküls im angeregten Zustand sehr hoch ist oder die Geschwindigkeit der strahlungslosen Deaktivierung niedrig in Relation zur Strahlungsgeschwindigkeit ist. Die Emission wird als Fluoreszenz bezeichnet, wenn die Emission nach der Lichtabsorption schnell zurückgeht $\left(10^{-9}-10^{-3} \mathrm{~s}\right.$.). Bei der Phosphoreszenz dagegen dauert die Emission nach der Absorption länger (charakteristischerweise einige Sekunden) an (z.B. bunte Lichtstäbe bei Veranstaltungen).

\section{Mechanismen der PDT-Wirkung}

Wird nun Gewebe mit Aminolävulinsäure (ALA) behandelt, dann werden speziell in neoplastischen Zellen Porphyrinmetaboliten synthetisiert (Abb.1). Wird nun ALA-behandeltes Gewebe Licht ausgesetzt, dann produzieren die angereicherten Porphyrinmetaboliten (im speziellen Protoporphyrin IX) (Abb. 2) eine photodynamische Reaktion. Es läuft ein zytotoxischer Prozess ab, der von der gleichzeitigen Anwesenheit von Licht und Sauerstoff abhängig ist (Abb. 3) [1]. 


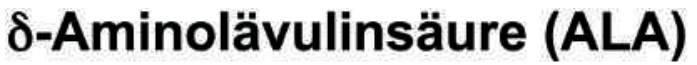

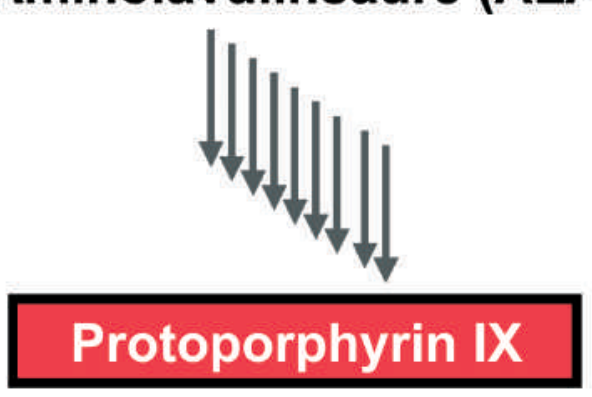

Abb. 1 Innerhalb der Porphyrinbiosynthese werden mehrere enzymatische Schritte durchlaufen, um aus ALA Porphyrinmetaboliten zu bilden.

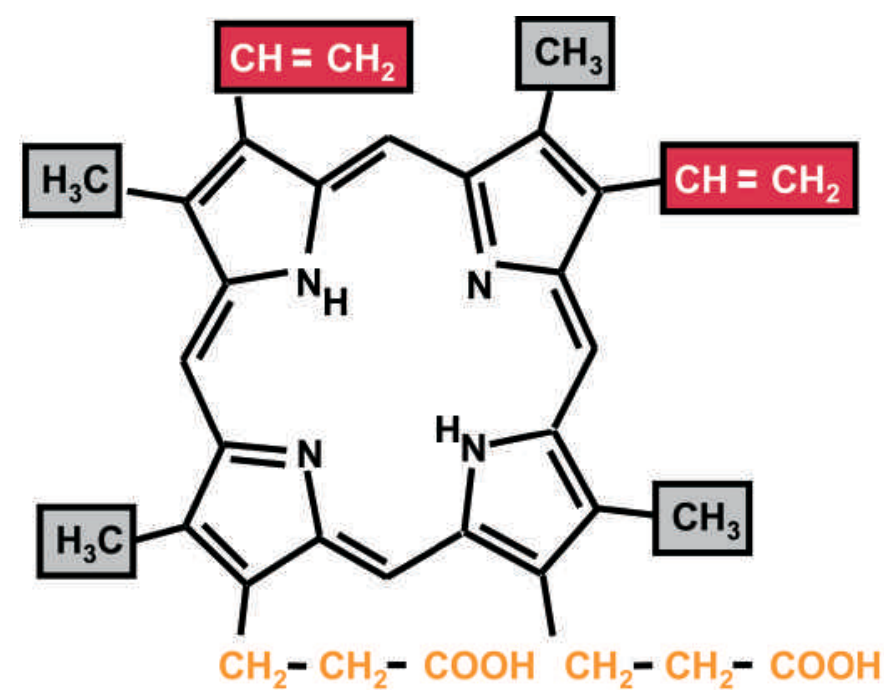

Abb. 2 Protoporphyrin ist ein Porphyrinmolekül mit sehr starker photosensibilisierender Eigenschaft. Das Molekül besteht aus einem Tetrapyrrolring und 2 Carboxylgruppen.

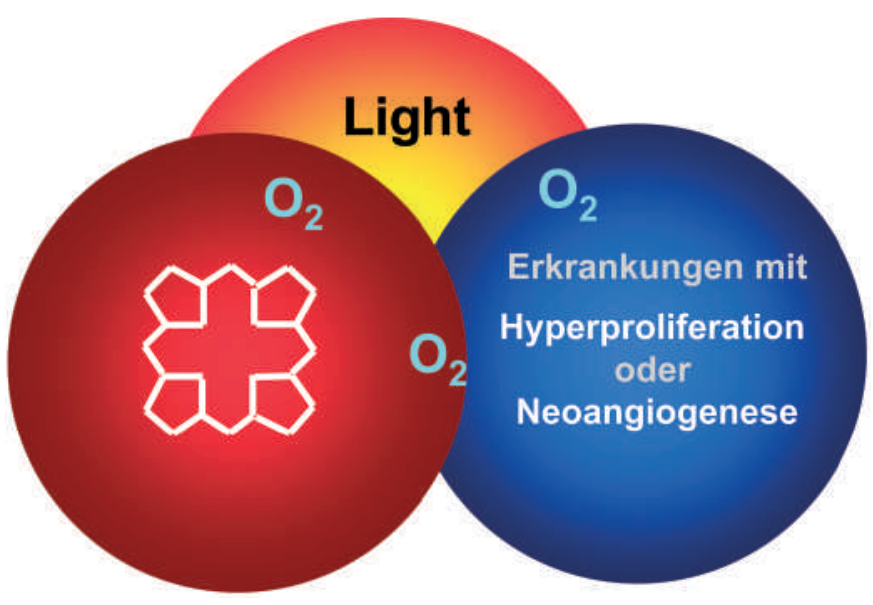

Abb. 3 Neben dem Tumorgewebe sind Sauerstoff und Licht für eine photodynamische Aktion entscheidend.
Der initiale Schritt des photosensibilisierenden Mechanismus ist die Absorption von Lichtphotonen durch den Sensitiser. Es entsteht ein Shift von seinem Grundzustand in den extrem instabilen angeregten Singulet-Level. Das angeregte Photosensibilisatormolekül fällt anschließend in seinen Grundzustand zurück, woraus die Emission von Licht in Form von Fluoreszenz resultiert (welche in der FD genutzt wird). Alternativ, geht der angeregte Photosensibilisator über den Weg des Intersystem crossing in den etwas stabileren Triplet-Zustand über. Die Interaktion des Triplet-Sensitizer mit den umliegenden Molekülen resultiert in zwei Arten photooxidativer Reaktionen (Abb. 4). Der Typ I involviert den Transfer von Elektronen oder Wasserstoffatomen, um Radikalformen des Photosensitizers oder dessen Substrate zu bilden (Abb. 5). Diese Intermediate können nun in Folge mit Sauerstoff reagieren, um Peroxide, Superoxidionen und Hydroxylradikale zu bilden, welche wiederum freie Radikalkettenreaktionen einleiten. Der Typ-II-Mechanismus wird durch Energietransferprozesse mit Sauerstoff im Grundzustand mediiert. Es folgt die Formation von Singulet-Sauerstoff und der Rückgang des Sensibilisators in seinen Grundzustand.

Der über den Typ-II-Weg erzeugte Singulet-Sauerstoff spielt die zentrale Rolle in der photodynamischen Zytotoxizität, da dieser höchst effektiv mit verschiedensten Biomolekülen interagiert. Als frühe Effekte nach PDT lassen sich mikroskopisch und biochemisch erkennbare Veränderungen an den Membranen von Zellen und Organellen beobachten. Lipidperoxidation und Protein-cross-linking verändern die Depolarisierung und inaktivieren die Membranenzyme. Die verstärkte Membranpermeabilität hemmt den Transport von Aminosäuren und Nukleosiden. Einige Stunden nach PDT entstehen Einbrechungen innerhalb der Plasmamembran, gefolgt von Zelllyse (Abb.6). Die PDT-vermittelte Zytotoxizität verursacht keine Primärschäden am Zellkern. Somit verspricht sich die PDT als Methode mit einem geringen Potenzial Mutationen zu generieren bzw. an Karzinogenese. Die Antwort auf die Photosensibilisierung umfasst die Aktivierung verschiedener Gene, die für Stress-Proteine, wie z. B. Heat-ShockProteine, Hämoxygenase, und glukoseregulierte Proteine kodieren. Weiterhin findet sich eine vorübergehende Induktion der Early Response Gene (z.B. c-fos, c-jun, c-myc) [2]. Die Phosphorylierung der p38 mitogenaktivierten Proteinkinase (MAPK) wird in einem vergleichbaren Maß nach PDT verstärkt. Die Effekte der PDT auf die MAPK im Sinne der Aktivierung der c-Jun$\mathrm{N}$-terminalen Kinase (JNK) und der p38 MAPKs sind vergleichbar denjenigen verursacht durch UV-Strahlung oder Hydrogenperoxid [2]. Neben dem direkten Zellschaden spielt die Schädigung des vaskulären Systems eine entscheidende Rolle in der Tumorzerstörung. Sauerstoffradikale schwächen die Barrierefunktion der Endothelzellen. Arterioläre Gefäßkonstriktion, venuläre Thrombusbildung und Blutstase verursachen einen indirekten Tumorzelltod durch nutritive Einbußen [3]. Die Degradierung der Phospholipide resultiert in dem Freisetzen von verschiedenen Entzündungsmediatoren. Akute Phase Proteine, Proteinasen, Peroxidasen, Komplementfaktoren und Zytokine werden aktiviert. Diese Entzündungssignale führen zur Anreicherung von Immuneffektorzellen wie beispielsweise neutrophilen Granulozyten und Makrophagen. 


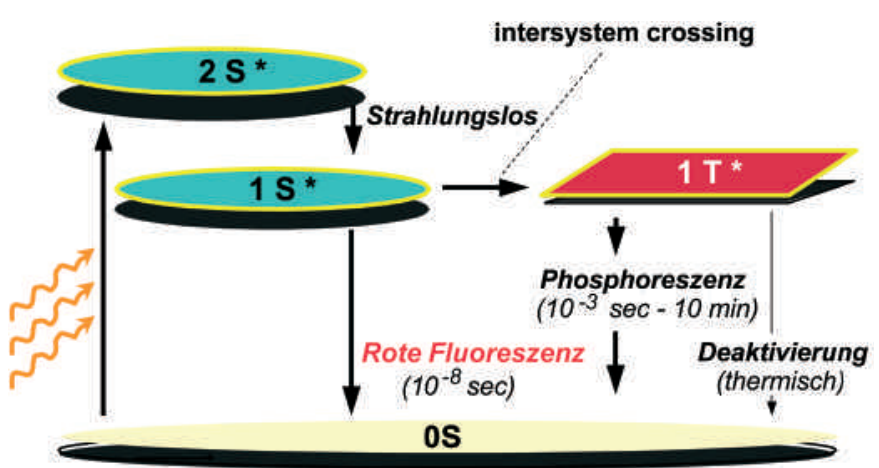

Abb. 4 Die verschiedenen Energieniveaus des Photosensibilisatormoleküls. Der aktivste Zustand ist der so genannte Triplet-Zustand 1T*.

Typ I

Reaktion mit Zellen

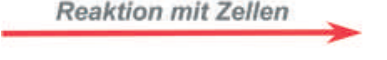

Freie Radikale

Oxidation oder Reduktion des PS

Typ II Via Oxygen

$\underset{\text { Energietransfer auf Sauerstoff }}{\longrightarrow}$ Singulet Oxygen PS wird zu os deaktiviert

Abb. 5 Die Energie des Photosensibilisators (1T*) im hochenergetischen Zustand kann über 2 Mechanismen zytotoxisch wirken.
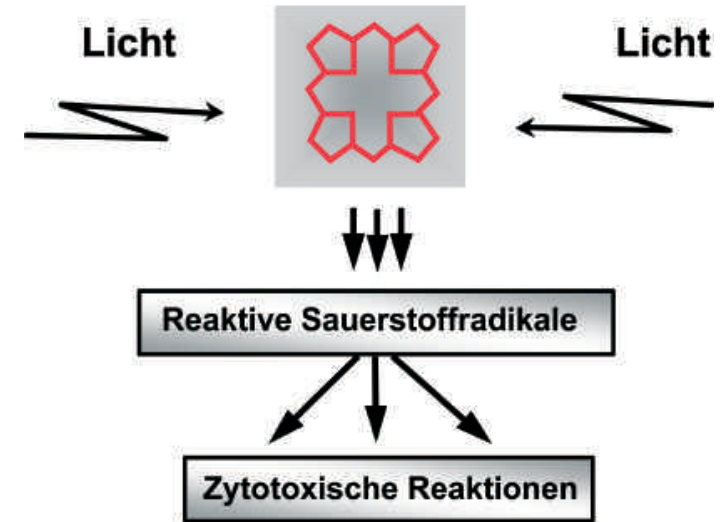

Abb. 6 Die reaktiven Sauerstoffspezies, die aufgrund einer photodynamischen Aktion entstehen, führen zum Zelltod.

\section{Photosensibilisatoren}

Der Sensibilisator, der zur topischen PDT am häufigsten eingesetzt worden ist, ist ALA (Abb. 7). In den vergangenen fünf Jahren wurde darüber hinaus die Effektivität von weiteren topisch applizierbaren Substanzen (Porphyrinvorstufen) untersucht. Hier ist vor allem der Methylester der ALA (MALA oder MAOP = methyl-[5-amino-4-oxopentanoate]) zu nennen. ALA wird zumeist mit einer Salbe vermischt. Vorteile einer Salbe - im Vergleich zur Creme - sind der bessere Halt auf der Hautoberfläche und eine optimierte perkutane Penetration. Dagegen bietet die Creme den Vorteil der stärkeren Hydrophilität und somit eine homogenere und leichtere Vermischung mit der ALA. Allgemein

\section{$\delta$-Aminolävulinsäure (ALA)}

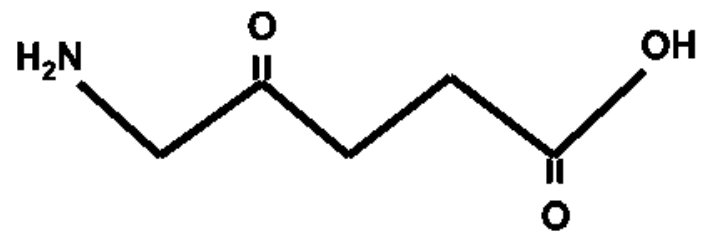

Abb. 7 Chemische Struktur der ALA.

sollte darauf geachtet werden, eine Grundlage zu wählen, die möglichst wenig Konservierungs- und Duftstoffe enthält. Insbesondere haben sich Basis-Salben, z.B. Neribas ${ }^{\circledR}$ Salbe oder die wasserhaltige hydrophile Salbe (DAB 10; Unguentum emulsificans aquosum) als wirksam erwiesen. Weiterhin zeichnete sich in den vergangenen Jahren ab, dass MAOP $\left(\right.$ Metvix $^{\circledR}=16 \%$ ALAMethylester in Creme; Galderma) selektiver als ALA neoplastisches Gewebe mit Porphyrinen anreichert [4]. Tumoren können sich in der FD somit noch deutlicher von der gesunden Haut abgrenzen lassen. Für die PDT bedeutet dies, dass die tumorumliegende Haut weniger belastet wird. Für die FD sollten ca. 0,2 g Creme oder Salbe $/ \mathrm{cm}^{2}$ mit ALA-Konzentrationen zwischen 10 und $20 \%$ (entspricht ca. $20-40 \mathrm{mg} \mathrm{ALA} / \mathrm{cm}^{2}$ ) bzw. 16\% MAOP ( = Metvix ${ }^{\circledR}$ ) appliziert werden. Bei der Behandlung von disseminiert lokalisierten aktinischen Keratosen am Kapillitium wäre prinzipiell eine großflächige Applikation der ALA/MAOP-Mixtur sinnvoll. Aufgrund des relativ hohen Materialverbrauchs, empfiehlt es sich, hier bevorzugt die suspekten bzw. tastbaren Hautbereiche inklusive eines Sicherheitsabstandes zu behandeln. Das entsprechende Haut- bzw. Tumorareal wird mit einer Folie (z.B. Tegaderm $^{\circledR}, 3 \mathrm{M}$, oder Frischhaltefolie) bedeckt, fixiert (mit z.B. Fixomull ${ }^{\circledR}$ ) und einem lichtundurchlässigen Verband abgedeckt, um die Penetration der Substanz zu verstärken und ein Photobleaching zu vermeiden. Dieser Lichtschutz ist nur für Läsionen im Gesicht, am Kopf oder an den Händen relevant. Ein Basalzellkarzinom am Unterarm beispielsweise wird anschließend durch die Kleidung bedeckt. Auch im Falle einer großflächigen Applikation, zum Beispiel bei disseminierten aktinischen Keratosen am gesamten Kapillitium, ist die okklusive Behandlung nicht unbedingt erforderlich. Es genügt ein (in)direkter Lichtschutz, z. B. abgedunkelter Raum oder ein Hut. Nach einer Einwirkungszeit von drei bis vier Stunden werden der Verband und die Salbenrückstände entfernt. In einem komplett abgedunkelten Raum erfolgt die Beleuchtung des Gewebes mit einer Wood-Licht-Lampe. Das gewonnene Fluoreszenzbild kann anschließend fotografisch festgehalten werden. Dies ist insbesondere dann empfehlenswert, wenn z.B. der Verlauf einer Tumortherapie dokumentiert werden soll und später Vergleichsaufnahmen zu einer aktuellen Fluoreszenz vorliegen sollten [1].

\section{Lichtquellen}

Bezüglich der Bestrahlungslampen wird der Markt derzeitig von verschiedensten Geräten überströmt. Dies liegt sicherlich auch daran, dass die Lichtindustrie nach dem Rückgang des Interesses an konventionellen Bestrahlungsgeräten wie UV-A oder UVB-311nm-Kabinen, wieder einen innovativen Ansatz hat, um an der Entwicklung medizinischer Geräte teilzuhaben. Die in 
Deutschland zuerst (1993) bereitgestellten Geräte waren die PDT 700 und in Folge „der große Bruder“, die PDT 1200 von Waldmann (Lichtquellen, die rotes Licht von 570-750 nm emittieren). 1995 entwickelte die Fa. Saalmann den Prototypen des jetzigen Fluolight-Greenlight-Komplexes. Dieser beinhaltet eine TandemEinheit aus hochenergetischem Wood-Licht zur Fluoreszenzdetektion und einer effektiven Grünlichtquelle (um 545 nm). Im Zuge der Einführung von ALA als Medikament stellte Schering den von DUSA entwickelten Kerastik vor. Dieser besteht aus einem 2-Kompartment Plastikröhrchen. In einem Teil ist die ALA als Pulver lokalisiert, in dem anderen das Lösungsmittel. Da die Zulassungsstudien mit blauem Licht, der BLU-U durchgeführt worden waren, wurde die Zulassung dieses Produktes in Deutschland auch an die Benutzung von blauem Licht gekoppelt. Das war der Grund, warum Waldmann das Modell PDT $450 \mathrm{~L}$, eine der BLU-U ähnlichen Lampe, konzipierte, um diese auf dem hiesigen Markt anbieten zu können. Im Detail hat das Gerät die Form einer Haube, die erlaubt, das gesamte Kapillitium und zusätzlich auch Teile des Gesichtes in einer Sitzung zu beleuchten. Limitierend bei diesem Konzept ist derzeitig, dass sich Schering mit der europäischen Zulassung des Kerastik zurückhält und deswegen auch die Technologie der blauen Lampen wenig Fuß fasst. Somit gab es in den vergangenen Monaten oder Jahren für interessierte Kolleginnen und Kollegen nur die Möglichkeit die ALA oder den ALA-Ester als Chemikalie einzukaufen und vom Apotheker als Magistralrezeptur in einer Salben- oder Cremegrundlage anmischen zu lassen. Durch die Verfügbarkeit des von medac entwickelten Arzneimittels ALA wurde die Zugängigkeit zur Substanz wesentlich erleichtert. Dennoch ist die Unsicherheit unter den Dermatologen nach wie vor recht groß, wenn es darum geht, die PDT als Routinetherapie in den Praxisalltag mit zu integrieren. Seit wenigen Monaten ist nun ein Fertigpräparat auf dem Markt. Der von der norwegischen Firma Photocure international getestete ALA-Methylester (MAOP) wird in Deutschland von Galderma als Metvix ${ }^{\circledR}$ vertrieben. Photocure hat auch die Entwicklung einer neuer Lampentechnologie eingeleitet. Die so genannte Aktilite emittiert Schmalbandlicht um $634 \mathrm{~nm}$, das durch einzelne LED generiert wird. Aktilite wird in zwei verschiedenen Größen angeboten, wobei sich die Modelle lediglich in deren Bestrahlungsfläche nicht aber in der Bestrahlungsleistung unterscheiden. Waldmann hat aktuell das Konzept der LED-Technologie übernommen und vertreibt diese in Form der Omnilux PDT.

Aufgrund des zunehmenden Interesses an der PDT wird immer wieder die Frage geäußert, welche Lampe denn nun für die PDT die geeignetste sei. Wir denken, dass es an dieser Stelle äußerst wichtig ist, eine klare Stellungnahme zu geben, um Verunsicherungen zu vermeiden. Zum besseren Verständnis ist es wichtig, das Absorptionsspektrum der Porphyrinmoleküle zu betrachten (Abb. 8). Hier sehen Sie die Wellenlängenbereiche, bei denen die Porphyrine viel bzw. am meisten Licht aufnehmen können. Es zeigen sich im Kurvenverlauf Gipfel bei 405 (UV bis blau), 505 (blau), 540 (grün), 580 (gelb) und $635 \mathrm{~nm}$ (rot). Das bedeutet, dass z. B. blaues Licht (im Wood-Licht-Bereich) von den Porphyrinen sehr stark absorbiert wird. Dagegen wird Licht um die 450 $\mathrm{nm}$ bzw. $600 \mathrm{~nm}$ relativ gering von den Porphyrinen aufgenom-

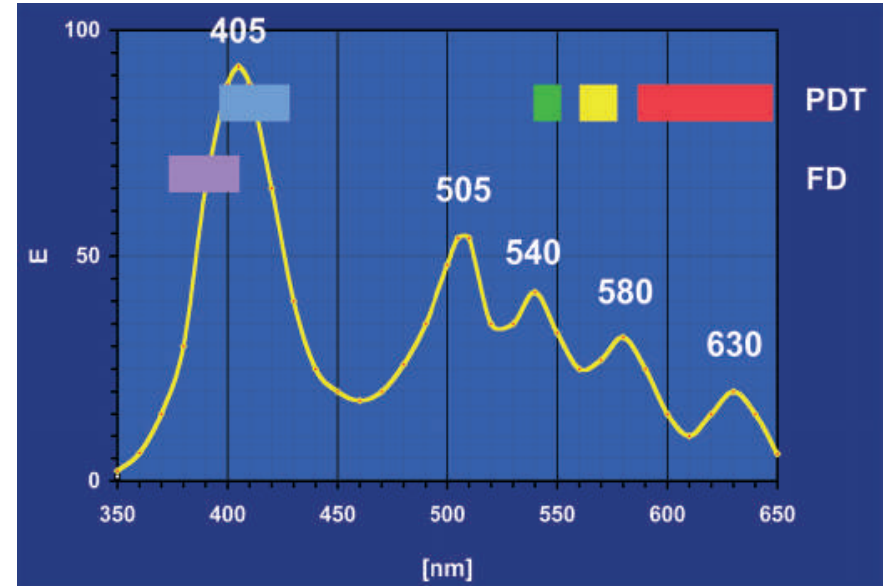

Abb. 8 Absorptionsspektrum der Porphyrinmoleküle. Die Porphyrine nehmen hauptsächlich Wellenlängen im Übergangsbereich vom UVALicht zum sichtbaren Licht auf. Weiterhin finden sich 4 Gipfel im Bereich höherer Wellenlängen von absteigender Intensität. Zur FD wird violettes Licht verwendet. Es sei hier angemerkt, dass in der PDT dagegen grünes oder rotes Licht verwendet wird. Hier finden sich zwar geringere Absorptionsausbeuten; Licht vom grünen bzw. roten Bereich hat aber die Eigenschaft tiefer (als die violette Strahlung) in das Gewebe einzudringen.

men. In den vergangenen Jahren war es Ziel der Lichtindustrie in Zusammenarbeit mit wissenschaftlichen Arbeitsgruppen, das geeignetste Licht für die PDT zu finden. Hierbei wurde versucht Wellenbereiche zu wählen, die die Porphyrine selektiv anregen, ohne uneffektives Licht mit einzuschließen. Wichtig in diesem Zusammenhang ist zu wissen, dass Licht hoher Wellenlängen (z.B. rotes Licht um $635 \mathrm{~nm}$ ) tiefer in Gewebe eindringt als z.B. blaues Licht um $405 \mathrm{~nm}$. Somit lassen sich tiefer gelegene Tumorzellen mit rotem Licht effektiver behandeln als mit blauem Licht. Diese Diskussion um den Einsatz verschiedener Wellenlängen in der PDT relativiert sich, wenn man berücksichtigt, dass die topische Applikation der ALA eine homogene Sensibilisierung mit Porphyrinen von maximal 1 bis $2 \mathrm{~mm}$ Tiefe hervorruft. Diese Tiefe wird mit jedem Licht zwischen 400 und 700 $\mathrm{nm}$ erreicht.

\section{Literatur}

${ }^{1}$ Fritsch C, Ruzicka T. Fluorescence diagnosis and photodynamic therapy in dermatology. Altas and handbook. Wien, New York: Springer Verlag, 2003

${ }^{2}$ Klotz LO, Fritsch C, Briviba K, Tsacmacidis N, Schliess F, Sies H. Activation of JNK and p38 but not ERK MAP kinases in human skin cells by 5-aminolevulinate-photodynamic therapy. Cancer Res 1998; 58: 4297-4300

${ }^{3}$ Dellian M, Abels C, Kuhnle GE, Goetz AE. Effects of photodynamic therapy on leucocyte-endothelium interaction: differences between normal and tumour tissue. Br J Cancer 1995; 72: 1125-1130

${ }^{4}$ Soler AM, Warloe T, Berner A, Giercksky KE. A follow-up study of recurrence and cosmesis in completely responding superficial and nodular basal cell carcinomas treated with methyl 5-aminolaevulinatebased photodynamic therapy alone and with prior curettage. British Journal of Dermatology 2001; 145: 467-471 